\title{
Apigenin inhibits HeLa sphere-forming cells through inactivation of casein kinase $2 \alpha$
}

\author{
JIE LIU $^{1 *}$, XIAO-CHENG CAO ${ }^{2 *}$, QIAO XIAO ${ }^{2}$ and MEI-FANG QUAN ${ }^{2}$ \\ ${ }^{1}$ Department of Gynaecology and Obstetrics, First Affiliated Hospital of University of South China, Hengyang, Hunan 421001; \\ ${ }^{2}$ Laboratory of Medicine Engineering, Medical College, Hunan Normal University, Changsha, Hunan 410013, P.R. China
}

Received December 20, 2013; Accepted June 9, 2014

DOI: $10.3892 / \mathrm{mmr} .2014 .2720$

\begin{abstract}
The protein kinase casein kinase 2 (CK2) has been implicated in stem cell maintenance and its aberrant activation has been demonstrated in several types of cancer, including cervical cancer. In the present study, it was demonstrated that the sphere-forming cells (SFCs) of HeLa cell lines exhibited self-renewal capacity, indicating that they possessed the properties of cervical cancer stem-like cells. HeLa-derived SFCs exhibited a higher level of CK2 $\alpha$ protein, compared with the parental cells. Apigenin, a dietary flavonoid, led to a dose-dependent inhibition of the self-renewal capacity and the protein expression of CK $2 \alpha$ in HeLa-derived SFCs. Furthermore, forced overexpression of CK $2 \alpha$ resulted in a decrease in the inhibition of CK2 $\alpha$ expression and the self-renewal capacity induced by apigenin in HeLa-derived SFCs. These results suggested that apigenin inhibits the self-renewal capacity of HeLa-derived SFCs through downregulation of CK $2 \alpha$ expression.
\end{abstract}

\section{Introduction}

Several studies have demonstrated that flavonoids are preventive in cancer $(1,2)$. The most common nonmutagenic flavonoid, 4',5,7-trihydroxyflavone (apigenin), has demonstrated marked effects in inhibiting cancer cell growth in vitro and in vivo (2-4). Apigenin has also been found to possess anti-inflammatory and antioxidant properties $(5,6)$ and inhibit tumor cell invasion, metastasis (7), mitogen-activated protein kinases and downstream oncogenes (8). Our previous study demonstrated that apigenin was able to affect the number of glioma stem-like cells (GSLCs) derived from U251 cells (9)

Correspondence to: Ms. Mei-Fang Quan, Laboratory of Medicine Engineering, Medical College, Hunan Normal University, 371 Tongzipo Road, Yeulu, Changsha, Hunan 410013, P.R China E-mail: quanmeifang@yeah.net

\section{${ }^{*}$ Contributed equally}

Key words: cervical cancer, cervical cancer stem cells, apigenin, casein kinase $2 \alpha$, self-renewal
However, the effect of apigenin on the self-renewal capacity of cervical cancer stem-like cells (CCSLCs) remains to be elucidated.

The protein kinase casein kinase 2 (CK2) is a highly conserved serine/threonine kinase with a broad spectrum of substrates. CK2 is a multifunctional protein kinase that has been demonstrated to be involved in almost every aspect of cell proliferation and survival (10-13). The expression and activity of CK2 are frequently elevated in cancer cells, including cervical cancer $(14,15)$. Previous studies by Zhang et al demonstrated that the function of CK2 $\alpha$ is involved in the activation of Hedgehog (Hh) and Notch pathways and in the maintenance of cancer stem cell properties $(16,17)$. Whether or not targeting CK2 by the selective CK2 kinase inhibitor apigenin leads to self-renewal inhibition of cervical cancer stem-like cells remains to be elucidated.

The present study was performed to examine whether apigenin inhinited the self-reneweal capactiy of sphere-forming cells (SFCs) of the cervical cancer HeLA cell line and its underlying mechanisms, which aimed to assess the possibility for its use in the treatment of human cervical cancer by targeting cancer stem cells.

\section{Materials and methods}

Reagents. Apigenin was obtained from Sigma (St. Louis, MO, USA) and was dissolved in dimethyl sulfoxide to a final concentration of $0.1 \%$ in media without causing cytotoxicity. Anti- $\beta$-actin and CK $2 \alpha$ antibodies were obtained from Calbiochem (La Jolla, CA, USA).

Cell culture. The HeLa human cervical cell line was maintained in Dulbecco's modified Eagle's medium (DMEM; Gibco, Carslbad, CA, USA) supplemented with $10 \%$ fetal bovine serum (Gibco), 100 units/ml penicillin (Gibco) and $100 \mu \mathrm{g} / \mathrm{ml}$ streptomycin (Gibco). In all experiments, the cells were maintained at $37^{\circ} \mathrm{C}$, in a $5 \% \mathrm{CO}_{2}$ and $95 \%$ air atmosphere. All the experiments were performed on cultures that were $70 \%$ confluent. The present study was approved by the ethics committee of Hunan Normal University (Changsha, China).

Tumorsphere culture. Single cell suspensions were suspended at a density of 5,000 cells $/ \mathrm{ml}$ in serum-free DMEM/F12 
supplemented with $100 \mathrm{IU} / \mathrm{ml}$ penicillin, $100 \mu \mathrm{g} / \mathrm{ml}$ streptomycin, $20 \mathrm{ng} / \mathrm{ml}$ human recombinant epidermal growth factor, $10 \mathrm{ng} / \mathrm{ml}$ human recombinant basic fibroblast growth factor, 2\% B27 supplement without vitamin A and 1\% N2 supplement (Invitrogen Life Technologies, Carlsbad, CA, USA) and seeded into ultra low attachment 6-well plates (Corning Inc., Corning, NY, USA). Suspension cultures were continued for 6 days until tumorspheres were formed. In order to propagate spheres in vitro, the sphere cells were collected by centrifugation ( $1,000 \mathrm{~g}$ for $5 \mathrm{~min})$, dissociated into single-cell suspensions and cultured to permit the regeneration of spheres. Third-generation spheres were used for all subsequent experiments.

To investigate the percentage of single cells capable of regenerating new spheres, cells were plated at a density of 1,000 cells $/ \mathrm{ml}$ in a 6 -well plate in order to obtain new spheres. The total number of tumor spheres was counted after 6 days of culture. Sphere formation efficiency was calculated using the following formula: (Total number of spheres formed / total number of live cells seeded) x 100 .

Limiting dilution analysis. The third-generation spheres were dissociated, as described above, and 100 cells were plated in $150 \mu 1$ of growth medium in a 96-well culture plate to obtain a single cell per well. Growth medium $(20 \mu \mathrm{l})$ was added to each well every 3 days. The number of clonal tumor spheres in each 96-well culture plate was evaluated after 6 days of culture.

MTT assay. SFCs from the HeLa cell line and the parental cells were seeded in 96-well plates precoated with Matrigel (Gibco-BRL) at a density of 5,000 cells per well. Cells were exposed to increasing concentrations $(10,20$ and $40 \mu \mathrm{mol} / \mathrm{l})$ of apigenin. After 48 h, MTT reagent (Sigma) was added to each well according to the manufacturer's instructions. Absorbance was measured at $570 \mathrm{~nm}$ using an automated microplate reader (Bio-RAD 550; Bio-Rad, Hercules, CA, USA).

Overexpression of the CK2 $\alpha$ protein. The pcDNA3.1-CK2 $\alpha$ or control pcDNA3.1-LacZ plasmid vectors were transfected into HeLa cells or the SFCs of HeLa cells $(0.5 \mu \mathrm{g} / \mathrm{ml}$ in a 24 -well plate $)$ using Lipofectamine 2000 transfection reagent (Invitrogen Life Technologies), according to the manufacturer's instructions. The cells were resuspended in complete medium (DMEM supplemented with $10 \%$ fetal bovine serum; Gibo-BRL) for $48 \mathrm{~h}$. The cells were harvested and western blotting was performed using mouse monoclonal antibodies against $\beta$ actin and CK2 $\alpha$.

Western blot analysis. Western blot analysis was performed, as previously described (18). Cells were lysed by incubating in lysis buffer for $20 \mathrm{~min}$ at $4^{\circ} \mathrm{C}$. The protein concentration was determined using the Bio-Rad assay system (Bio-Rad, Hercules, CA, USA). Total proteins were fractionated using SDS-PAGE and transferred onto a polyvinylidene fluoride membrane (Millipore, Billerica, MA, USA). Signals were detected using an ECL Advance western blot analysis system (Amersham Pharmacia Biotech, Inc., Piscataway, NJ, USA).

Statistical analysis. Statistical analysis and database management was performed using SPSS (version 15.0) software
(SPSS, Inc., Chicago, IL, USA). Data are expressed as the mean \pm standard deviation. Multiple group comparisons were made using one-way analysis of variance and pairwise comparison was performed using the least squares difference method. $\mathrm{P}<0.05$ was considered to indicate a statistically significant difference.

\section{Results}

Sphere formation and self-renewal in the HeLa cell line. HeLa cells were plated in stem cell-conditioned culture medium in 6-well plates at a density of 10,000 cells/well, which enabled the formation of discrete colonies. Under these conditions, cells grew as non-adherent, three-dimensional sphere clusters. The HeLa cella maintained in DMEM medium supplemented with $10 \%$ fetal bovine serum (monolayer adhere growth cells) and anchorage-independent spheres formed in the HeLa cell line are shown in Fig. 1A and Fig 1B, respectively. The spheres were passaged after 6 days once they had reached $\sim 50 \mu \mathrm{m}$ in diameter. The HeLa sphere-derived cells were serially passaged for $>12$ generations, indicating that HeLa sphere-derived cells were fully capable of self-renewal in vitro.

To determine the percentage of CCSLCs in HeLa third-generation spheres, a limiting dilution assay was used to examine the ability of single cells from third-generation spheres to produce new spheres. After 6 days of culture, $31 \%$ of the single cells had generated new spheres (Fig. 1C). By contrast, a lower percentage of single cells derived from the HeLa cells could regenerate spheres when compared with the single cells derived from third-generation spheres (Fig. 1D). These results demonstrated that a considerable percentage of single cells derived from third-generation spheres were self-renewing cells that were able to be expanded and maintained in culture as tumor spheres.

Apigenin inhibits the proliferation and self-renewal capacity of the HeLa sphere-derived cells. It has been reported that cancer stem cells have the characteristics of extensive proliferation and apigenin has been demonstrated to inhibit the proliferative activity of glioma cancer stem-like cells (9). In the present study, the MTT results demonstrated that apigenin $(10,20$ and $40 \mu \mathrm{mol} / \mathrm{l})$ preferentially inhibited the proliferation of SFCs derived from HeLa cells (Fig. 2A), suggesting that apigenin is able to preferentially suppress the proliferative ability of CCSLCs.

Apigenin (10, 20 and $40 \mu \mathrm{mol} / \mathrm{l})$ reduced the number of spheroids formed in the SFCs of HeLa cells in a concentration-dependent manner (Fig. 2B). These results suggest that apigenin can suppress the self-renewal capacity of CCSLCs.

Apigenin downregulates the protein expression of $C K 2 \alpha$ in HeLa sphere-derived cells. Previous studies have reported that CK2 $\alpha$ is involved in the activation of the Hh and Notch pathways and is associated with the maintenance of cancer stem cell properties $(16,17)$. The present study aimed to compare the status of CK2 $\alpha$ protein expression in parental cells and in SFCs. The results demonstrated that the expression of CK $2 \alpha$ was higher in the SFCs than in the parental cells (Fig. 3A). In addition, CK2 $\alpha$ expression in SFCs was downregulated by apigenin (Fig. 3B). 
A

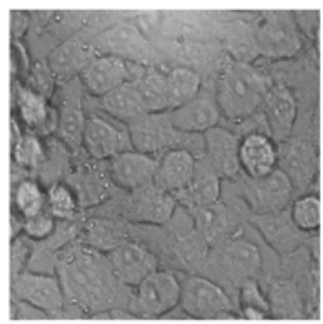

C

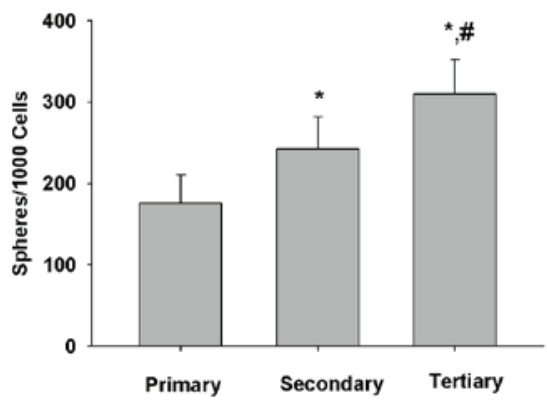

B

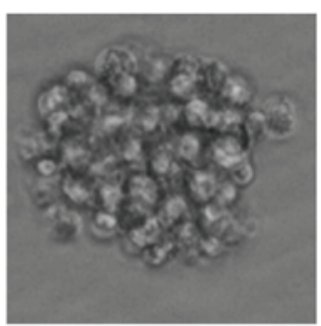

D

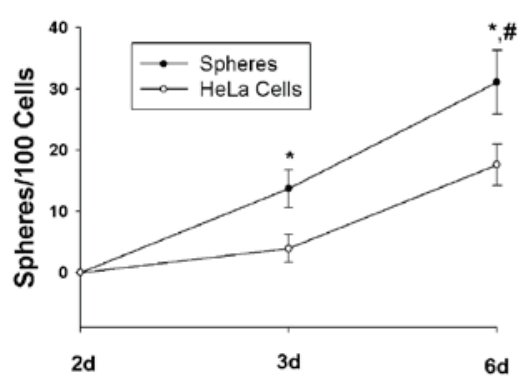

Figure 1. Sphere formation and self-renewal in the HeLa cell line. (A) Monolayer adhere growth of HeLa cells in Dulbecco's modified Eagle's medium supplemented with $10 \%$ fetal bovine serum. (B) Anchorage-independent spheres formed in HeLa cells maintained in stem cell-conditioned culture medium. (C) Single cells were dissociated from tumor spheres and plated at a density of 1,000 cells $/ \mathrm{ml}$ to allow the regeneration of new spheres. The total number of primary, secondary and tertiary spheres were counted. ${ }^{*} \mathrm{P}<0.05$, vs. the number of primary spheres formed by 1,000 parental HeLa cells; ${ }^{*} \mathrm{P}<0.05$, vs. the number of secondary spheres formed by 1,000 single cells dissociated from primary spheres. (D) Number of spheres formed in 100 single cells derived from third-generation spheres was compared with that of single cells derived from the parental HeLa cells. " $\mathrm{P}<0.05$, vs. the number of spheres formed by 100 parental HeLa cells after 3 days of culture; ${ }^{*} \mathrm{P}<0.05$, vs. the number of spheres formed by 100 parental HeLa cells after 3 days of culture and after 6 days of culture.

A

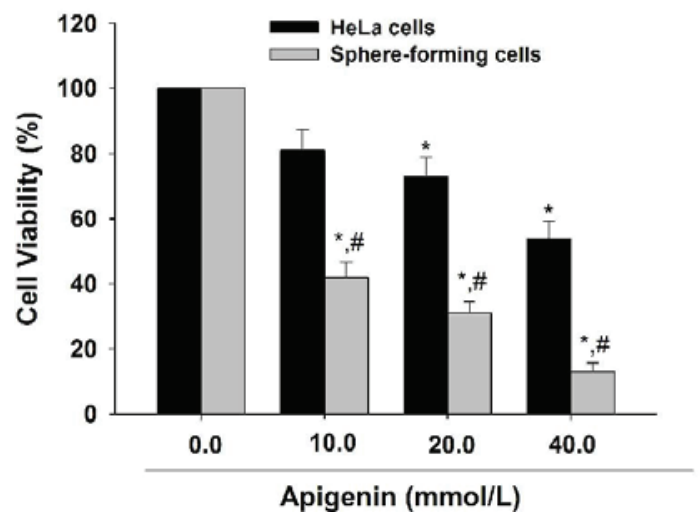

B

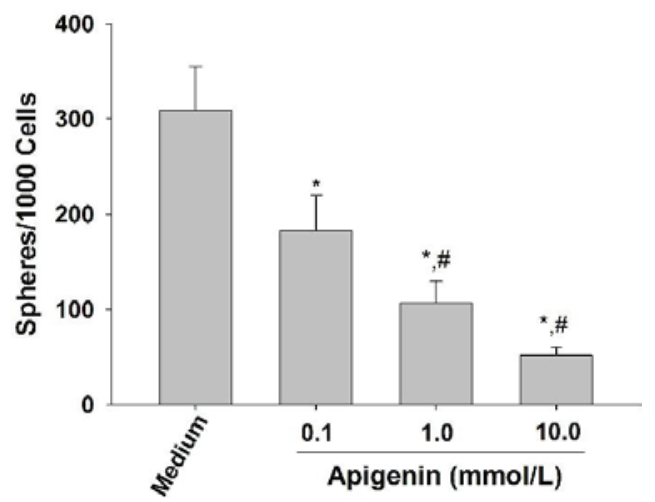

Figure 2. Apigenin inhibits the proliferation and self-renewal capacity of HeLa SFCs. (A) SFCs and parental HeLa cells were treated with increasing concentrations of apigenin for $48 \mathrm{~h}$. The anti-proliferative effect of apigenin was measured using an MTT assay. ${ }^{*} \mathrm{P}<0.05$, vs. non-treated SFCs or parental HeLa cells; ${ }^{\#} \mathrm{P}<0.05$, vs. parental HeLa cells treated with corresponding concentrations of apigenin. (B) Apigenin decreased the number of spheroids formed in HeLa SFCs in a concentration-dependent manner. ${ }^{*} \mathrm{P}<0.05$, vs. the untreated group; ${ }^{*} \mathrm{P}<0.05$, vs. the $0.1 \mathrm{mmol} / 1$ apigein-treated group. SFCs, sphere forming cells; DMSO, dimethyl sulfoxide; medium, untreated.

Overexpression of $C K 2 \alpha$ attenuates the inhibitory effects of apigenin on the self-renewal capacity of HeLa sphere-derived cells. Western blot analysis demonstrated that upregulation of CK2 $\alpha$ by pcDNA3.1-CK $2 \alpha$ transfection resulted in overexpression of the $\mathrm{CK} 2 \alpha$ protein in the parental HeLa cells (Fig. 4A). The results from the tumorsphere formation assay revealed that overexpression of CK2 $\alpha$ attenuated apigenin-induced downregulation of CK $2 \alpha$ protein expression in the HeLa SFCs (Fig. 4B) and also revealed that overexpression of the $\mathrm{CK} 2 \alpha$ protein increased the self-renewal capacity of HeLa cells (Fig. 4C). In addition, . It also partially reduced the inhibition of self-renewal of SFCs by apigenin (Fig. 4D). These results provided mechanistic evidence that apigenin-inhibited self-renewal was, in part, due to inactivation of CK $2 \alpha$ in SFCs derived from HeLa cells.

\section{Discussion}

The present study demonstrated that a natural dietary flavonoid, apigenin, inhibited the proliferation and self-renewal 
A

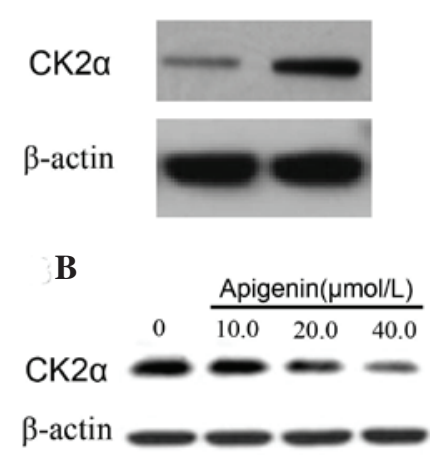

Figure 3. Apigenin decreases the protein expression of CK2 $\alpha$ in HeLa SFCs. (A) CK2 $\alpha$ expression was higher in SFCs than in the PC. (B) CK2 $\alpha$ expression in SFCs was downregulated by apigenin. SFCs, sphere-forming cells; PC, parental cells; CK2 $\alpha$, casein kinase $2 \alpha$.

capacity of HeLa sphere-derived cells and inhibited the protein expression of CK2 $\alpha$. The inhibition of the CK2-mediated self-renewal capacity in HeLa sphere-derived cells by apigenin may be the primary mechanism mediating the anticancer activities of apigenin.

Previous studies have reported that the function of CK $2 \alpha$ is involved in the activation of $\mathrm{Hh}$ and Notch pathways and in the maintenance of cancer stem cell properties $(16,17)$. In order to investigate the potential mechanism through which CK2 positively regulates the self-renewal capacity of CCSLSCs, the present study performed a tumorsphere formation assay in the parental HeLa cells following transfection with pcDNA3.1-CK $2 \alpha$. The results indicated that forced overexpression of CK2 $\alpha$ resulted in an increase in sphere formation rate in HeLa cells. Furthermore, apigenin, a small molecule CK $2 \alpha$ inhibitor, significantly inhibited sphere formation of HeLa sphere-derived cells. Taken together, these results suggest that $\mathrm{CK} 2 \alpha$ is important in facilitating the self-renewal capacity of CCSLCs. Further investigation is required to elucidate the precise mechanisms.

Zhao et al (19) found that apigenin inhibits proliferation and induces apoptosis in human multiple myeloma cells through the regulation of CK2, Cdc37 and Hsp90 expression. Feliciano et al (20) indicated that miR-125b is inversely correlated with glutamyl aminopeptidase and CK2 $\alpha$ expression in breast tumorigenesis and that $\mathrm{CK} 2 \alpha$ overexpression is associated with the presence and number of lymph node metastases. Our previous study demonstrated that apigenin preferentially inhibited the proliferation of GSLCs derived from U251 cells (9). The results of the present study demonstrated that apigenin significantly inhibited the proliferation and tumorsphere formation of HeLa sphere-derived cells. Furthermore, forced overexpression of CK $2 \alpha$ effectively attenuated the apigenin inhibited tumorsphere formation of HeLa sphere-derived cells. These results suggested that apigenin-inhibited self-renewal is partly due to the inactivation of CK $2 \alpha$ in SFCs derived from HeLa cells.

In conclusion, the present study demonstrated that CK2 is a positive regulator in the self-renewal of CCSLCs and apigenin, inhibiting the self-renewal capability, is involved in the downregulation of $\mathrm{CK} 2 \alpha$ protein expression. The
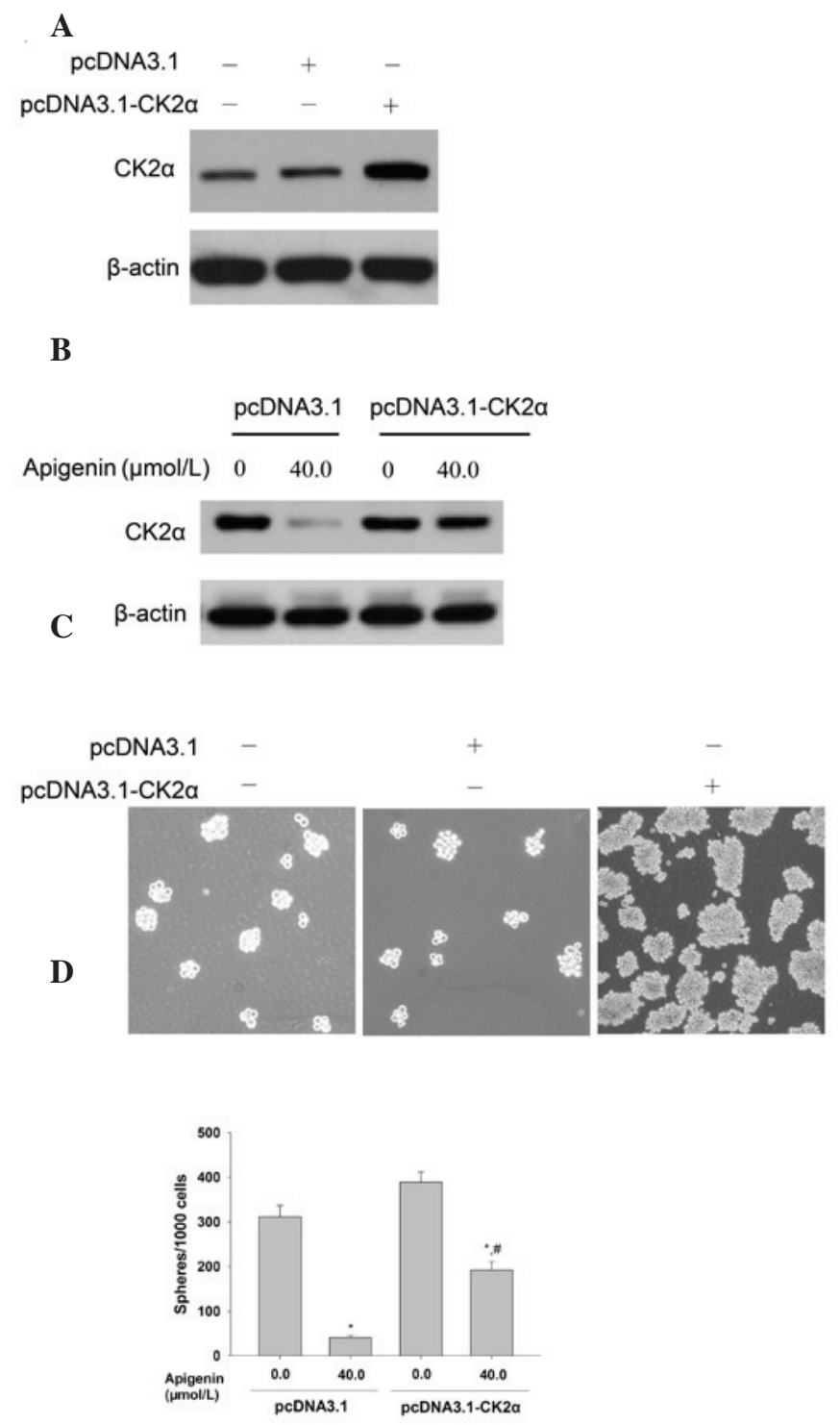

Figure 4. Overexpression of $\mathrm{CK} 2 \alpha$ attenuates the inhibitory effects of apigenin on the self-renewal capacity of HeLa sphere-forming cells. (A) pcDNA3.1-CK2 $\alpha$ transfection resulted in overexpression of the CK2 $\alpha$ protein in the parental HeLa cells. (B) Overexpression of the CK2 $\alpha$ protein increased the self-renewal capacity of HeLa cells. (C) Overexpression of CK2 $\alpha$ attenuated apigenin-induced downregulation of CK2 $\alpha$ expression in HeLa SFCs. (D) Overexpression of CK $2 \alpha$ reduced inhibition of self-renewal in HeLa SFCs induced by apigenin. ${ }^{*} \mathrm{P}<0.05$, vs. pcDNA3.1 transfected SFCs; ${ }^{\text {}} \mathrm{P}<0.05$, vs. $40 \mu \mathrm{mol} / 1$ apigenin-treated pcDNA3.1 transfected SFCs or pcDNA3.1-CK $2 \alpha$ transfected SFCs. SFCs, sphere forming cells; CK2 $\alpha$, casein kinase $2 \alpha$.

findings of the present study provide important evidence for the potential benefits of CK2 inhibitors in the treatment of human cervical cancer by targeting cancer stem cells.

\section{Acknowledgements}

The authors would like to thank Professor Jian-Guo Cao and Dr Xi-Yun Deng, Medical College, Hunan Normal University, (Changsha, Hunan, China) for their input into the scientific content of this study. The present study was supported by the Construct Program of the Key Discipline of Basic Medicine in Hunan. 


\section{References}

1. Birt DF, Hendrich S and Wang W: Dietary agents in cancer prevention: flavonoids and isoflavonoids. Pharmacol Ther 90: $157-177,2001$

2. Benavente-García $\mathrm{O}$ and Castillo J: Update on uses and properties of citrus flavonoids: new findings in anticancer, cardiovascular, and anti-inflammatory activity. J Agric Food Chem 56: 61856205,2008

3. Arango D, Morohashi K, Yilmaz A, Kuramochi K, Parihar A, Brahimaj B, Grotewold E and Doseff AI: Molecular basis for the action of a dietary flavonoid revealed by the comprehensive identification of apigenin human targets. Proc Natl Acad Sci USA 110: e2153-e2162, 2013

4. Oishi M,Iizumi Y,TaniguchiT,GoiW,MikiTandSakai T: Apigenin sensitizes prostate cancer cells to Apo2L/TRAIL by targeting adenine nucleotide translocase-2. PLoS One 8: e55922, 2013.

5. Pham H, Chen M, Takahashi H, King J, Reber HA, Hines OJ, Pandol S and Eibl G: Apigenin inhibits NNK-induced focal adhesion kinase activation in pancreatic cancer cells. Pancreas 41 : $1306-1315,2012$

6. Kim HK, Cheon BS, Kim YH, Kim SY and Kim HP: Effects of naturally occurring flavonoids on nitric oxide production in the macrophage cell line RAW 264.7 and their structure-activity relationships. Biochem Pharmacol 58: 759-765, 1999.

7. Raso GM, Meli R, Di Carlo G, Pacilio M and Di Carlo R: Inhibition of inducible nitric oxide synthase and cyclooxygenase-2 expression by flavonoids in macrophage J774A.1. Life Sci 68: 921-931, 2001.

8. Lindenmeyer F, Li H, Menashi S, Soria C and Lu H: Apigenin acts on the tumor cell invasion process and regulates protease production. Nutr Cancer 39: 139-147, 2001.

9. He J, Xu Q, Wang M, Li C, Qian X, Shi Z, Liu LZ and Jiang BH: Oral administration of apigenin inhibits metastasis through AKT/P70S6K1/MMP-9 pathway in orthotopic ovarian tumor model. Int J Mol Sci 13: 7271-7282, 2012.

10. Feng X, Zhou Q, Liu C and Tao ML: Drug screening study using glioma stem-like cells. Mol Med Rep 6: 1117-1120, 2012

11. Litchfield DW: Protein kinase CK2: structure, regulation and role in cellular decisions of life and death. Biochem J 369: 1-15, 2003.

12. Trembley JH, Wang G, Unger G, Slaton J and Ahmed K: Protein kinase CK2 in health and disease: CK2: a key player in cancer biology. Cell Mol Life Sci 66: 1858-1867, 2009.

13. Ahmad KA, Wang G, Unger G, Slaton J and Ahmed K: Protein kinase CK2 - a key suppressor of apoptosis. Adv Enzyme Regul 48 179-187, 2008.
14. Piazza FA, Ruzzene M, Gurrieri C, Montini B, Bonanni L, Chioetto G, Di Maira G, Barbon F, Cabrelle A, Zambello R, Adami F, Trentin L, Pinna LA and Semenzato G: Multiple myeloma cell survival relies on high activity of protein kinase CK2. Blood 108: 1698-1707, 2006.

15. Solares AM, Santana A, Baladrón I, Valenzuela C, González CA, Díaz A, Castillo D, Ramos T, Gómez R, Alonso DF, Herrera L, Sigman H, Perea SE, Acevedo BE and López-Saura P: Safety and preliminary efficacy data of a novel casein kinase 2 (CK2) peptide inhibitor administered intralesionally at four dose levels in patients with cervical malignancies. BMC Cancer 9: 146, 2009.

16. Perea SE, Baladron I, Garcia Y, Perera Y, Lopez A, Soriano JL, Batista N, Palau A, Hernández I, Farina H, Garcia I, Gonzalez L, Gil J, Rodriguez A, Solares M, Santana A, Cruz M, Lopez M, Valenzuela C, Reyes O, López-Saura PA, González CA, Diaz A, Castellanos L, Sanchez A, Betancourt L, Besada V, González LJ, Garay H, Gómez R, Gómez DE, Alonso DF, Perrin P, Renualt JY, Sigman H, Herrera L and Acevedo B: CIGB-300, a synthetic peptide-based drug that targets the CK2 phosphoaceptor domain. Translational and clinical research. Mol Cell Biochem 356: 45-50, 2011.

17. Zhang S, Long H, Yang YL, Wang Y, Hsieh D, Li W, Au A, Stoppler HJ, Xu Z, Jablons DM and You L: Inhibition of CK2 $\alpha$ down-regulates Notch1 signalling in lung cancer cells. J Cell Mol Med 17: 854-862, 2013.

18. Zhang S, Wang Y, Mao JH, Hsieh D, Kim IJ, Hu LM, $\mathrm{Xu} \mathrm{Z}$, Long H, Jablons DM and You L: Inhibition of CK2 $\alpha$ down-regulates Hedgehog/Gli signaling leading to a reduction of a stem-like side population in human lung cancer cells. PLoS One 7: e38996, 2012

19. Ren KQ, Cao XZ, Liu ZH, Guo H, Quan MF, Liu F, Jiang L, Xiang HL, Deng XY and Cao JG: 8-bromo-5-hydroxy-7-m ethoxychrysin targeting for inhibition of the properties of liver cancer stem cells by modulation of Twist signaling. Int J Oncol 43: 1719-1729, 2013.

20. Zhao M, Ma J, Zhu HY, Zhang XH, Du ZY, Xu YJ and Yu XD: Apigenin inhibits proliferation and induces apoptosis in human multiple myeloma cells through targeting the trinity of CK2, Cdc37 and Hsp90. Mol Cancer 10: 104, 2011.

21. Feliciano A, Castellvi J, Artero-Castro A, Leal JA, Romagosa C, Hernández-Losa J, Peg V, Fabra A, Vidal F, Kondoh H, Y, Cajal S and Lleonart ME: miR-125b acts as a tumor suppressor in breast tumorigenesis via its novel direct targets ENPEP, CK2- $\alpha$, CCNJ and MEGF9. PLoS One 8: e76247, 2013. 